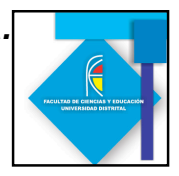

\title{
UNA PROPUESTA DE FORMALIZACIÓN DEL PRINCIPIO DE CONSERVACIÓN DE LA ENERGÍA DESDE LA PERSPECTIVA DE MAYER
}

\author{
A PROPOSAL TO FORMALIZE THE PRINCIPLE OF \\ CONSERVATION OF ENERGY FROM THE OUTLOOK MAYER
}

\author{
Christian Camilo Agudelo Restrepo ${ }^{1}$ \\ Óscar David Palacio Muñoz, Julián ${ }^{2}$ \\ Andrés Londoño Zuleta ${ }^{3}$ \\ Yirsen Aguilar Mosquera ${ }^{4}$
}

\section{Resumen}

En los análisis realizados en investigaciones, es notoria la dificultad que presentan algunos estudiantes en la conceptualización y aplicación del principio de conservación de la energía, entre otros se destaca la no distinción entre el concepto de trabajo y el concepto de energía, lo que lleva a una circularidad cuando se trata de explicar cualquier fenómeno aplicando estos conceptos. Como propuesta alternativa, se plantea una recontextualización de dicho principio, a partir del análisis histórico epistemológico de la obra (Fuerzas de la Naturaleza Inorgánica, Mayer, 1973), por considerar que su particular manera de formalizar el Principio de Conservación de la Energía, no solo permite una resignificación de los conceptos involucrados, sino que además, permite plantear rutas didácticas alternativas para la enseñanza de de este Principio de Conservación.

La propuesta de recontextualización planteada se estructura a partir de la construcción y aplicación, de una serie de instrumentos (estos surgen de los planteamientos de Mayer) que indagan sobre ideas alternativas $y$, buscan el uso de nuevos modelos explicativos del fenómeno. Según el análisis de los resultados obtenidos, se concluye que, la energía, al verse bajo la relación causa-efecto, se logra inducir a través de la convertibilidad y la conservación de la misma. Dada las diferentes dificultades en la enseñanza, se hizo necesario el diseño de estrategias didácticas fundamentadas en la historia y la epistemología que se considera pueden viabilizar la enseñanza y el aprendizaje de la física.

Palabras clave: Energía, conservación, transferencia, degradación, convertibilidad, causalidad.

\footnotetext{
${ }^{1}$ Estudios Culturales Sobre las Ciencias y su EnSEÑAnZA -ECCE-Facultad de Educación, Universidad de Antioquia. Medellín, Colombia. kcamilo29@gmail.com,

${ }^{2}$ Estudios CULTURALES SOBRE LAS CIENCIAS y SU ENSEÑANZA -ECCE-Facultad de Educación, Universidad de Antioquia. Medellín, Colombia. palacio2805@gmail.com,

${ }^{3}$ ESTUdios CULTURALES SOBRE LAS CiENCIAS Y SU ENSEÑANZA -ECCE-Facultad de Educación, Universidad de Antioquia. Medellín, juanloz007@yahoo.com

${ }^{4}$ Estudios CUlturales Sobre las Ciencias y su EnSeÑanza -ECCE-Facultad de Educación, Universidad de Antioquia. Medellín, , yirsena@yahoo.es
} 


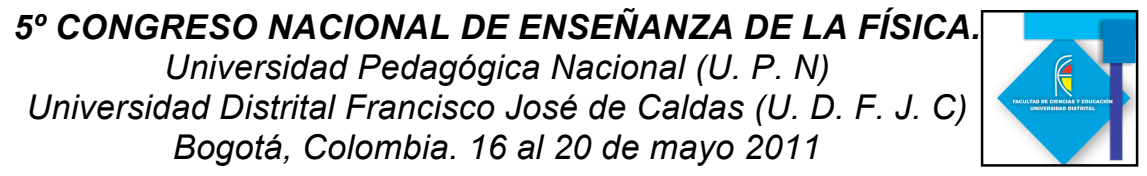

\begin{abstract}
In the analysis made in research, is notoriously difficult, for several students in the conceptualization and implementation of the principle of conservation of energy, among other highlights failure to distinguish between the concept of work and the concept of energy, which leads to circularity when trying to explain any phenomenon by applying these concepts. As an alternative proposal raises a recontextualization of that principle, by analyzing historical epistemology of the work (Forces of Inorganic Nature, Mayer, 1973), arguing that their particular way of formalizing the Principle of Conservation of Energy, no only allows a redefinition of the concepts involved, but also allows us to suggest alternative routes to teaching teaching this Principle of Conservation.

The proposal is structured recontextualization raised from the construction and application of a series of instruments (these arise from the approach of Mayer) to inquire about alternative ideas and seek the use of new models that explain the phenomenon. According to the analysis of the results, we conclude that energy, to be under the cause-effect is achieved through induction and maintenance of convertibility of it. Given the various difficulties in teaching, it became necessary to design teaching strategies based on the history and epistemology can be considered viable teaching and learning of physics.
\end{abstract}

Keywords: Energy conservation, transfer, demotion, convertible causality.

\title{
El Principio de Conservación de la Energía en el contexto de la enseñanza
}

En los análisis realizados en investigaciones se puede resaltar algunos problemas que presentan los estudiantes en cuanto a la conceptualización de la energía y su conservación. En este sentido se resalta la confusión entre trabajo y esfuerzo (Driver y Warrington, 1985), la dificultad para identificar trabajo y la energía (Duit ,1984; Driver y Warrington, 1985), materialización de la energía (Duit, 1987 a; Salomón, 1983), considerar que la energía puede gastarse (Kesidou y Duit, 1993) o almacenarse (Salomón, 1985), confundir las formas de energía con sus fuentes (Carr y Kirkwood, 1998; Salomón, 1995), atribuirle al cuerpo la energía potencial y no a la interacción entre los cuerpos (Solbes y Matín, 1991), ignorar la variación de la energía interna (Van Huls y Van den Berg, 1993), asignar un carácter sustancial al calor (Albert, 1978; Erickson, 1979, 1980), confundir la cantidad de calor y la temperatura (Arnold, 1994). Y no comprenden los esquemas de transformación, conservación, transferencia y degradación de la energía (Duit, 1981, 1984).

En virtud de lo anterior, ¿cómo plantear, en el contexto de la enseñanza, un proceso de formalización del Principio de Conservación de la Energía desde un enfoque histórico epistemológico, a partir de los planteamientos de Robert Mayer?

Recontextualización del Conservación de la energía desde la perspectiva de Robert Mayer 


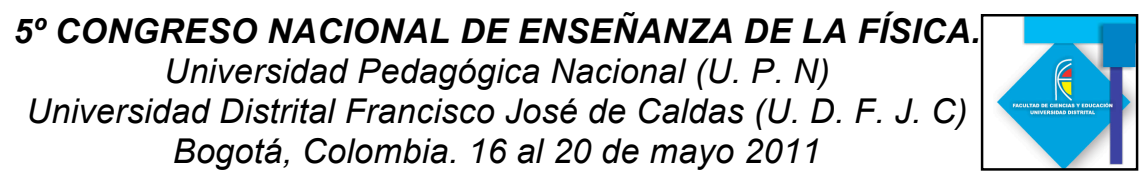

\section{Establecimiento condiciones iníciales}

Para Mayer (1973) formalizar el Principio de Conservación de la Energía, parte de la formulación del principio de causalidad, indestructibilidad de la materia y la convertibilidad de los fenómenos como condiciones iníciales en la formalización del Principio de Conservación de la Energía. Estas condiciones iníciales las aborda a partir de una conceptualización sobre la materia, fuerza (energía), movimiento y calor.

En el particular modo de Mayer ver el mundo físico, se plantean dos tipos de causas: las que son ponderables (materia) y las imponderables (fuerzas). La primera hace alusión a una característica cuantitativa que sólo es posible identificar a través de propiedades como peso y volumen. En éste sentido la materia es entendida en términos de indestructibilidad al igual que la fuerza. La segunda, a diferencia de la primera, se le otorga lo desconocido, lo hipotético. Lo que hasta donde la experiencia indicaba en aquel momento no es posible convertir materia en fuerza o viceversa. Aquí debe entenderse la fuerza no en el sentido newtoniano, sino que en términos de lo que hoy se conoce como energía.g

\section{Principio de Indestructibilidad}

Para llegar a comprender el fenómeno de la Conservación de la Energía, desde éste principio, se parte del siguiente razonamiento, considerando Mayer la energía como causa (principio causa eaquat effectum), a la causa inicial la denota con la letra c la cual produce un efecto e, concluye que $\mathbf{c}=\mathbf{e}$. Si después de producir el efecto $\mathbf{e}$, aún permanece la causa c en todo o en parte, debe haber otros efectos, correspondientes a la causa que aún queda. Y sí e es entonces causa, a su vez, de otro efecto $\mathbf{f}$, se tiene que $\mathbf{e}=\mathbf{f}$, por tanto $\mathbf{c}=\mathbf{e}=\mathbf{f} . . .=\mathbf{c}$. En esta cadena de causas y efectos, resulta evidente por la naturaleza de la igualdad, que un término o una parte de él, nunca pueden ser igual a cero" (Mayer, 1973, p. 277).

De este planteamiento, se desprenden aspectos bien interesantes: en primer lugar la causalidad como una función en donde la interdependencia de los fenómenos es el factor importante. En segundo lugar, como consecuencia de lo anterior, la energía puede ser asumida como causa de otros fenómenos o de otro efecto, que desencadena en una serie de consecuencias que la contienen en su totalidad o parcialmente. En el caso de contenerla parcialmente, debe pensarse en otras manifestaciones, pero en ningún caso será aniquilada o reducida a cero. En este sentido, se puede decir que, es justamente la consideración de la indestructibilidad de la materia y de las fuerzas lo que le permite a Mayer asumir la indestructibilidad de la energía y en consecuencia la conservación de la energía.

\section{Principio de Convertibilidad}

El Principio de Convertibilidad desde esta perspectiva causal, permite considerar las causas como generadoras de unos efectos, que a su vez, son considerados causas creadoras de otros efectos, esto es: 


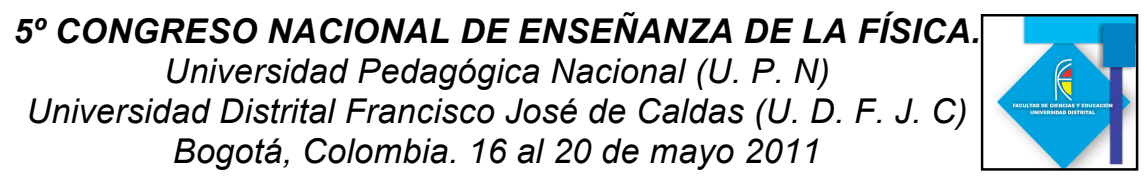

"si la causa $\boldsymbol{C}$ ha producido un efecto $\boldsymbol{e}$, entonces, por lo tanto, $\boldsymbol{C}$ ha dejado de existir convirtiéndose en e; si después de la producción de $\boldsymbol{e}, \boldsymbol{C}$ subsistiera aún, en todo o en parte, entonces a esta causa subsistente debería corresponder un efecto adicional; luego el efecto de $\boldsymbol{C}$ debería ser mayor que e $(\boldsymbol{C}>\boldsymbol{e})$, lo que es contrario al supuesto $\boldsymbol{C}=\boldsymbol{e}$. Por lo tanto, como $\boldsymbol{C}$ se convierte en $\boldsymbol{e}$, y a la vez éste se convierte en $\boldsymbol{f}$, etc. Debemos considerar estas magnitudes como las diferentes formas de manifestación de un sólo y mismo objeto" (Mayer, 1973, p. 277).

Bajo el principio de indestructibilidad, la causa es igual al efecto; pero dentro del principio de convertibilidad se contempla la posibilidad de que la causa no se convierta totalmente en su respectivo efecto, sino que de modo parcial, lo que resta de la causa, genera efectos adicionales rompiendo con la igualdad de la ecuación. Es en éste sentido, que los efectos de la causa inicial superan al efecto. La combinación de estas propiedades, le permitió a Mayer afirmar que las causas son objetos cuantitativamente indestructibles y cualitativamente convertibles, ya que obedecen a expresiones propias de un objeto. Tal reflexión, reafirma la característica imponderable de las causas y en últimas de la energía.

\section{Implicaciones didácticas}

Abordar la enseñanza de la física desde una histórico epistemológica, permite a los docentes de ciencia, establecer propuestas de enseñanza coherentes que permiten la construcción y comprensión holística de los conceptos, analizando las circunstancias que hacen posible situarlos en el contexto científico puesto que se examinan los contextos de producción. Estos aspectos, sin duda alguna, permiten, en la enseñanza y en el aprendizaje de la ciencia, una indagación más crítica y coherente con las explicaciones y modelos que son aceptados en el contexto científico. De este modo se subsanan algunas dificultades frente a la enseñanza, de la física puesto que se permite la solución de ciertas problemáticas de orden conceptual, como lo es el de Conservación de la Energía.

Bajo estas circunstancias, el pensar y reflexionar la física a la luz de la historia y la epistemología posibilita posturas didácticas que se concretan en propuestas que facilitan la comprensión y explicación de los fenómenos.

Plantear una formalización del principio de conservación de la energía desde la perspectiva de Mayer, implica comprender la interrelación entre objetos ponderables e imponderables desde una relación determinada por el Principio de Causalidad, que da origen a la convertibilidad entre las diferentes manifestaciones de un mismo objeto y por consiguiente a la indestructibilidad de la causa inicial o su conservación, es decir, una perspectiva de causalidad que permite explicar una situación física desde la interdependencia de los fenómenos.

Consecuente con lo anterior se asume que, la energía sólo es posible de ser identificada en la medida en que ésta adopta diferentes formas debidas a un proceso de convertibilidad de fenómenos. Desde esta perspectiva, el Principio de Conservación de la Energía posibilita, en el contexto de la enseñanza, una alternativa para abordar los fenómenos mecánicos.

La particular forma de Mayer llevar a cabo sus experimentaciones, le permitió establecer hipótesis sobre el fenómeno de la conservación de la energía, ya que realizo experimentos de tipo mental y físico, los cuales fueron determinantes para la organización de sus ideas. Es de esta manera como la experimentación permite establecer un vinculo con las teoría y 


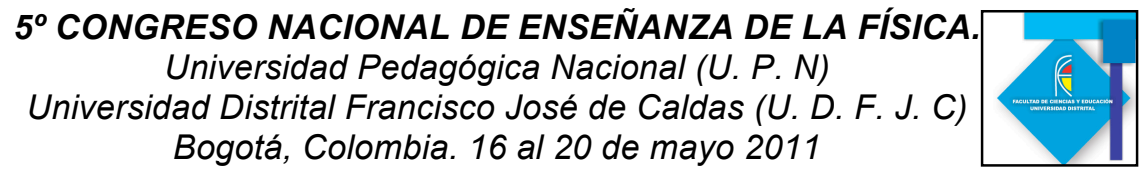

las observaciones realizadas, permitiendo la generación de nuevas formulaciones y evidenciar las ya planteadas.

Finalmente, teniendo en cuenta las consideraciones anteriores, es licito plantear que comprender el Principio de Conservación de la Energía, parte de entender el fenómeno desde la convertibilidad, la cual posibilita identificar las formas en las cuales puede transformarse la energía, aludiendo a un proceso en el cual la energía se conserva; es decir, parte por considerar causas iníciales como generadoras de unos efectos, que pueden desencadenar en otros efectos, que al finalizar el proceso tiene como resultado que los efectos sean iguales a la causa que los originó.

\section{Referencias bibliográficas}

Driver, R. y Warrington, L. (1985). Students' use of the principle of energy conservation in problem situations. Physics Education, 20, pp. 171-176.

SOLBES, J. y MARTÍN, J. (1991). Análisis de la introducción del concepto de campo. Revista Española de Física, 5, pp. 34-40.

Solbes, J. y Tarín, F. (1998). Algunas dificultades en torno a la conservación de la energía. Enseñanza de las ciencias, 16(3), 387-397.

Furió-Gómez Cristina, Solbes Jordi y Furió-Mas Carles. (2007). La historia del primer principio de la termodinámica y sus implicaciones didácticas. Revista. Eureka. Enseñanza y. Divagación Científica. 4(3), pp. 461-475.

Mayer, R. J. Onthe forces of inorganic nature. Reprinted from R. B. Linsay, Men of Physics, Oxford, 1973, pp. 277- 283. 